\title{
A Simple Jerky Dynamics, Genesio System
}

\author{
Ömür Umut, Serpil Yaşar \\ Department of Mathematics, Faculty of Arts \& Sciences, Abant Izzet Baysal University, Bolu, Turkey \\ Email: umut_o@ibu.edu.tr, say.maths@gmail.com
}

Received October 31, 2012; revised December 10, 2012; accepted December 19, 2012

\begin{abstract}
The third order explicit autonomous differential equations named as jerk equations represent an interesting subclass of dynamical systems that can exhibit many major features of the regular and chaotic motion. In this paper, we show that an algebraically simple system, the Genesio system can be recast into a jerky dynamics and its jerk equation can be derived from one-dimensional Newtonian equation. We also investigate the global dynamical properties of the corresponding jerk system.
\end{abstract}

Keywords: Genesio System; Algebraically Simple Systems; Jerky Dynamics; Newtonian Jerky Dynamics; Dynamical Properties

\section{Introduction}

The term jerk [1], i.e., the third derivative of displacement, $\dddot{x}$, has attracted some attention because of its relevance to the theory of chaos [2-11]. Some papers appeared in response to a question [2] posed by Gottlieb concerning simple jerk functions which may lead to chaotic phenomena. Sprott $[3,4]$ found several simple nonlinear jerk functions which gave strange attractor for appropriate choices of equation parameters and initial conditions. Linz $[5,6]$ introduced the idea and conditions for Newtonian jerky dynamics, derivable by differentiation of a (one-space dimension) Newtonian equation of motion for $\ddot{x}$, and analyzed the jerky dynamics for onevariable obtained from several familiar autonomous systems of three simultaneous first-order ordinary differential equations which are known to have chaotic solutions. He also allowed for the possibility of a memory or temporal history integral term in the force function. Coincidentally, Maccari [7] had considered such generalized oscillators, with nonlocal force terms, which obeyed an integro-differential equation and which were equivalent to an autonomous third-order nonlinear differential equation. His interests there were in periodic and quasi-periodic solutions.

As jerky dynamics can be considered a subclass of three-dimensional dynamical systems an interesting question [5] is which three-dimensional systems are equivalent to jerky dynamics. In [8], a class of three-dimensional nonlinear dynamical systems is studied which can be transformed into jerky dynamics. Most of the models of minimal chaotic dynamics considered in [8] belong to this class and can be transformed into jerky dynamics.
The transformations used have the restriction that the variable in the scalar differential equation is the same as the system. A consequence is that a linear transformation is sometimes not possible; the resulting transformation is nonlinear. In [9], it is shown that by removing this restriction, these models can be transformed to jerky dynamics via an affine transformation.

In [8], Eichhorn et al. used the method of Gröbner bases and showed that fifteen of Sprott's chaotic flows [4] can be recast into a jerk form. They also showed that these fifteen models, Sprott's minimal chaotic flow [4] and the Rössler toroidal model [10] can be arranged into seven classes (referred as $J D_{1}$ to $J D_{7}$ ) of jerky dynamics as a hierarchy of quadratic jerk equations with increasingly many terms as seen in Table 1. Such a classification provides simple means to compare the functional complexity of different systems and also demonstrate the equivalence of cases not otherwise apparent. In a subsequent study, Eichhorn et al. [11] examined the simplest cases of $J D_{1}$ and $J D_{2}$ in more detail and identified the regions of parameter space over which they exhibit chaos.

In this paper, we show that the Genesio system can be recast into a jerky dynamics by an affine transformation and the resulting form belongs to class $J D_{2}$. Moreover it is derived from one-dimensional Newtonian equation that is, it is a Newtonian jerky dynamics. Furthermore we investigate the global dynamics of that jerk equation and also show that it shares the common route to chaos as systems in class $J D_{2}$.

\section{Jerky Dynamics}

Consider the class of systems which can be written as a 
Table 1. Basic classes of dissipative jerky dynamics.

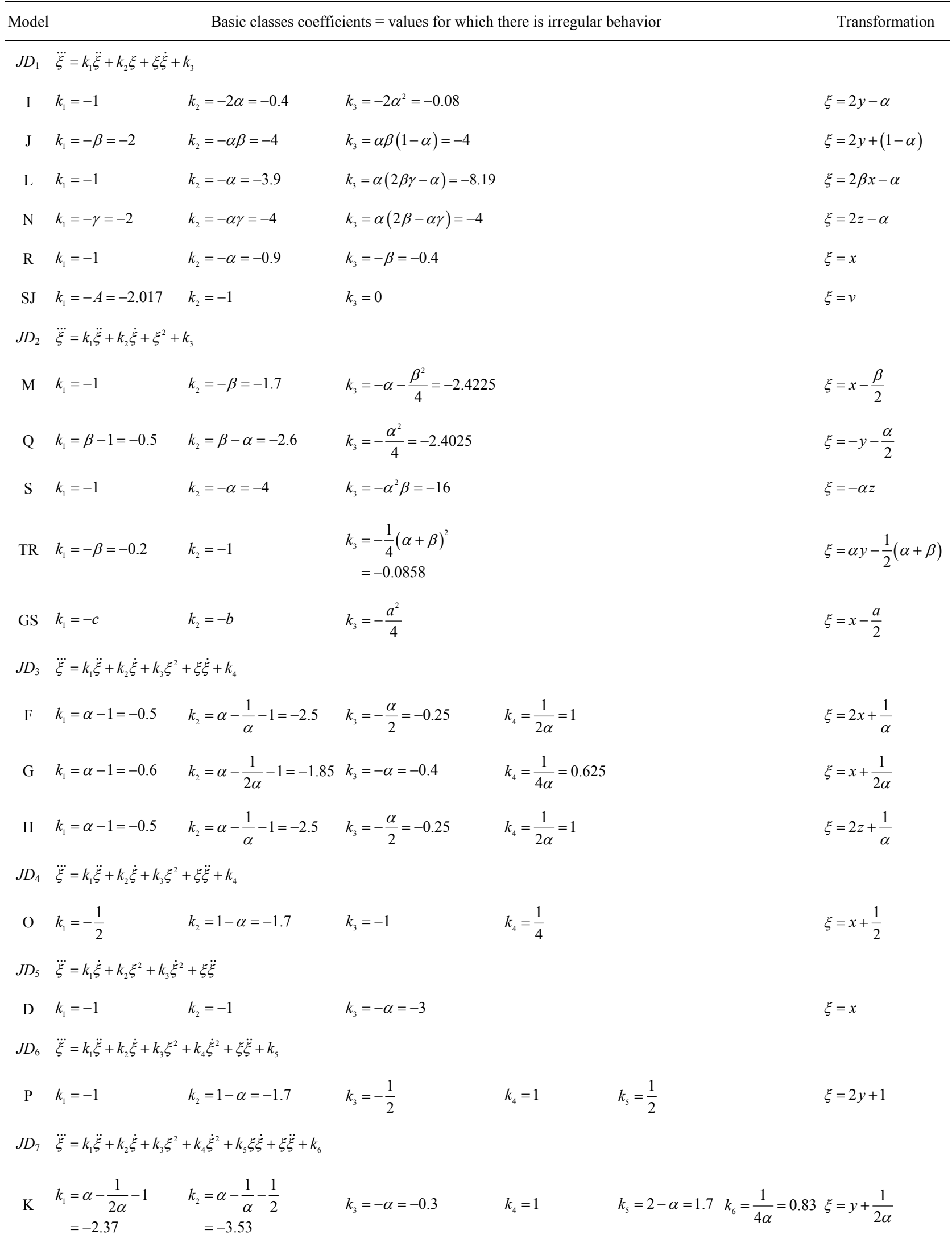


scalar ordinary differential equations:

$$
\xi^{(n)}=f\left(\xi, \dot{\xi}, \ddot{\xi}, \cdots, \xi^{(n-1)}\right)
$$

where $n$ is the order and $\xi^{(n)}$ denotes the $n$th derivative of the scalar state variable $\xi$. Clearly an $n$th order scalar ODE can be written as a system of $n$ first order ODEs. On the other hand, the following systems of equations can be rewritten as a scalar ODE:

$$
\dot{\boldsymbol{x}}=\left(\begin{array}{ccccc}
0 & 1 & & & \\
\ddots & \ddots & & & \\
& & & 0 & 1 \\
p_{0} & p_{1} & \cdots & & p_{n-1}
\end{array}\right) \boldsymbol{x}+\left(\begin{array}{c}
0 \\
\vdots \\
0 \\
f(x)
\end{array}\right) .
$$

For third order $(n=3)$ scalar ODEs, where $\xi$ is position, $\dddot{\xi}$ is the change in acceleration which is generally called the jerk, and the resulting dynamics are called jerky dynamics. In [8], a class of three-dimensional dynamical systems is considered whose members are topologically conjugate to jerky dynamics.

Theorem 2.1 Consider a three-dimensional system of the form

$$
\dot{\boldsymbol{x}}=A \boldsymbol{x}+\boldsymbol{n}(\boldsymbol{x})
$$

where $\boldsymbol{x} \in \mathbb{R}^{3}, A \in \mathbb{R}^{3 \times 3}$ is a matrix with constant coefficients $a_{i j}(i, j=1,2,3)$ and $\boldsymbol{n}(\boldsymbol{x})=\left(n_{1}(\boldsymbol{x}), n_{2}(\boldsymbol{x}), n_{3}(\boldsymbol{x})\right)^{\mathrm{T}}$ a three-dimensional vector solely nonlinear functions in $x$, $y, z$ that are twice differentiable and do not contain additive constants. If

$$
a_{12} n_{2}(\boldsymbol{x})+a_{13} n_{3}(\boldsymbol{x})=f\left(x, a_{12} y+a_{13} z\right)
$$

and

$$
a_{12}^{2} a_{23}-a_{13}^{2} a_{32}+a_{12} a_{13}\left(a_{33}-a_{22}\right) \neq 0
$$

then the system is topologically conjugate to a jerky $d y$ namics via a state transformation.

The state transformation in Theorem 2.1 has the restriction that the state variable $\xi$ in the corresponding jerky dynamics $\dddot{\xi}=f(\xi, \dot{\xi}, \ddot{\xi})$ is equal to one of the state variables in Equation (2). It was shown that 16 out of 20 simple chaotic systems considered in [8] fall into this class and thus are equivalent to jerky dynamics. For some of these systems, the corresponding state transformations are necessarily nonlinear. This is due to the above restriction on the state transformation. In [9] it was shown that without this restriction, simple linear transformations can be found which transform these systems into jerky dynamics. In particular, the following result gives sufficient conditions under which an $n$-dimensional system is topologically conjugate to a scalar ODE via an affine state transformation.

Definition 2.1. Let $A$ be an $\mathrm{n}$ by n matrix and $\boldsymbol{b}$ be an $n$ by 1 vector. The pair $(A, \boldsymbol{b})$ is controllable if the matrix

$$
K=(A, \boldsymbol{b})=\left(\boldsymbol{b}|A \boldsymbol{b}| A^{2} \boldsymbol{b}|\cdots| A^{n-1} \boldsymbol{b}\right)
$$

is nonsingular. The matrix $K$ is called the controllability matrix.

Theorem 2.2. Consider the system

$$
\dot{\boldsymbol{x}}=A \boldsymbol{x}+\boldsymbol{b} f(\boldsymbol{x})+\boldsymbol{c}
$$

where $A$ is an $n$ by $n$ matrix, $\boldsymbol{b}, \boldsymbol{c}$ are $n$ by 1 vectors and $f$ is a real-valued function. If $(A, \boldsymbol{b})$ is controllable, then the system is topologically conjugate to a scalar ODE via an affine transformation.

It is possible that a dynamical system that is contained in the class specified by Equation (5) can be converted simultaneously into two or three jerky dynamics in different variables (the jerky dynamics in the certain variable is unique, if it exists).

To obtain dynamical systems of the class in Theorem 2.2 with two simultaneously existing jerky dynamics, e.g., in $x$ and $y$ one has to restrict the nonlinear function $n_{2}(\boldsymbol{x})$ such that it is only function of $y$, i.e., $n_{2}(\boldsymbol{x})=$ $n_{2}(y)$. This follows directly from Equation (2). In addition to the conditions (3) and (4)

$$
\begin{array}{r}
a_{12} n_{2}(y)+a_{13} n_{3}(\boldsymbol{x})=f_{1}\left(x, a_{12} y+a_{13} z\right) \\
a_{12}^{2} a_{23}-a_{13}^{2} a_{32}+a_{12} a_{13}\left(a_{33}-a_{22}\right) \neq 0
\end{array}
$$

that ensure the existence of the jerky dynamics in $x$, there are also corresponding constraints for the jerky dynamics in $y$ that read explicitly

$$
\begin{gathered}
a_{21} n_{1}(x)+a_{23} n_{3}(\boldsymbol{x})=f_{2}\left(y, a_{21} x+a_{23} z\right) \\
a_{23}^{2} a_{31}-a_{21}^{2} a_{13}+a_{21} a_{23}\left(a_{11}-a_{33}\right) \neq 0
\end{gathered}
$$

where $f_{1}$ and $f_{2}$ are functions of the indicated arguments. Any dynamical system of functional form (5) with $n_{2}(\boldsymbol{x})=n_{2}(y)$ that fulfills the conditions (6)-(9) can be recast into an equivalent jerky dynamics in its variables $x$ and $y$. For simultaneously existing jerky dynamics in two other variables one has to take into account permutations of variables and indices, respectively.

For dynamical systems that possess simultaneously three jerky dynamics, further constraints apply. Clearly, $n_{3}(\boldsymbol{x})=n_{3}(z)$ must hold. Furthermore, in addition to Equations (6)-(9) there is a third condition reading explicitly

$$
\begin{array}{r}
a_{31} n_{1}(x)+a_{32} n_{2}(y)=f_{3}\left(z, a_{31} x+a_{32} y\right) \\
a_{31}^{2} a_{12}-a_{32}^{2} a_{21}+a_{31} a_{32}\left(a_{22}-a_{11}\right) \neq 0
\end{array}
$$

If a jerky dynamics can be derived from one-dimensional Newtonian equation by taking its derivative with respect to time we call the dynamics Newtonian jerky. The following theorem [6], states under which conditions a jerky dynamics can be Newtonian jerky:

Theorem 2.3. Any jerky dynamics of the functional 
form

$$
\dddot{x}+p(x, \dot{x}) \ddot{x}+q(x, \dot{x})=0
$$

with $p$ and $q$ being differentiable and integrable functions of their arguments $x$ and $\dot{x}$, is Newtonian jerky.

In the qualitative theory of dynamical systems $[12,13]$ gradient systems play an interesting role. For these systems, one can rule out the existence of oscillatory solutions just by considering their vector fields. In particular, a dynamical system is a gradient system if its vector field results from the gradient of a scalar potential. In [6], it is shown that there is no elementary criterion that excludes periodic solutions in some classes of Newtonian jerky dynamics.

Theorem 2.4. Newtonian jerky dynamics are not gradient systems.

Looking at the functional form of a jerky dynamics, it is highly nontrivial to decide whether it can have chaotic solutions for some parameter ranges or not. For some subclasses of jerky dynamics one can derive a simple criterion under what circumstances aperiodic or chaotic solutions cannot appear. Consider the jerky dynamics (12) with

$$
q(x, \dot{x})=r(x, \dot{x}) \dot{x}-s(x, \dot{x})
$$

where $r$ and $s$ are functions of the indicated arguments and $r(x, \dot{x})$ fulfills the Schwarz condition $\partial_{x} p(x, \dot{x})=$ $\partial_{\dot{x}} r(x, \dot{x})$. As a consequence the jerky dynamics (12) can be rewritten as

$$
\dddot{x}+p(x, \dot{x}) \ddot{x}+r(x, \dot{x}) \dot{x}=s(x, \dot{x})
$$

or equivalently,

$$
\frac{\mathrm{d}}{\mathrm{d} t}\left[\ddot{x}+\int^{\dot{x}} p(x, \dot{x}) \mathrm{d} \dot{x}\right]=\frac{\mathrm{d}}{\mathrm{d} t} \int^{t} s(x, \dot{x}) \mathrm{d} \tau .
$$

Direct integration of Equation (15) yields

$$
\ddot{x}+\int^{\dot{x}} p(x, \dot{x}) \mathrm{d} \dot{x}=\int^{t} s(x, \dot{x}) \mathrm{d} \tau .
$$

This shows most clearly that the left-hand side of Equation (16) can be interpreted as an oscillator coupled to an internal driving mechanism or feedback (the righthand side of Equation (16)) that is an integral over the history of its motion. This fact has some consequences for the possible dynamics of the jerky system (15).

Theorem 2.5. [6] If 1) the oscillator on the left-hand side of Equation (16), $\ddot{x}+\int^{\dot{x}} p(x, \dot{x}) \mathrm{d} \dot{x}=0$, possesses only bounded solutions and 2) the integrand of memory term, $s(x, \dot{x})$, on the right-hand side of Equation (16) is either positive semi-definite or negative semi-definite for all $x$ and $\dot{x}$, then the jerky dynamics (15) cannot show chaotic behavior.

\section{Genesio System as Jerky Dynamics}

The Genesio system, which was proposed by Genesio and Tesi [14], is described by the following simple threedimensional autonomous system with only one quadratic nonlinear term:

$$
\dot{x}=y, \dot{y}=z, \dot{z}=-a x-b y-c z+x^{2}
$$

where $a, b, c$ are real parameters.

Theorem 3.1. The Genesio system (17) can be recast into a jerky dynamics, and the resulting form belongs to class $\mathrm{JD}_{2}$.

Proof. The Equation (17) can be written as

$$
\dot{\boldsymbol{x}}=A \boldsymbol{x}+\boldsymbol{b} f(\boldsymbol{x})+\boldsymbol{c}
$$

where

$$
\begin{gathered}
\boldsymbol{x}=\left(\begin{array}{l}
x \\
y \\
z
\end{array}\right), A=\left(\begin{array}{ccc}
0 & 1 & 0 \\
0 & 0 & 1 \\
-a & -b & -c
\end{array}\right), \\
\boldsymbol{b}=\left(\begin{array}{l}
0 \\
0 \\
1
\end{array}\right), \boldsymbol{c}=\left(\begin{array}{l}
0 \\
0 \\
0
\end{array}\right), f(x)=x^{2} .
\end{gathered}
$$

The matrix $K=(A, \boldsymbol{b})=\left(\begin{array}{ccc}0 & 0 & 1 \\ 0 & 1 & -c \\ 1 & -c & c^{2}-b\end{array}\right)$ is nonsingular since $\operatorname{det}(\boldsymbol{K})=-1 \neq 0$. So, by Definition $2.1 \boldsymbol{K}$ is the controllability matrix and the pair $(A, \boldsymbol{b})$ is controllable. Hence the Genesio system can be recast into a jerky dynamics via an affine transformation by Theorem 2.2.

Application of the invertible transformation $\left\{\begin{array}{l}\dot{x}=y \\ \dot{y}=z\end{array}\right.$ to the Equation (17) yields

$$
\dddot{x}=-c \ddot{x}-b \dot{x}-a x+x^{2} .
$$

Using the linear and invertible transformation

$$
\xi=x-\frac{a}{2}, \dot{\xi}=\dot{x}, \ddot{\xi}=\ddot{x}
$$

and then replacing $\xi$ by $x$ we write Equation (19) as

$$
\dddot{x}=-c \ddot{x}-b \dot{x}+x^{2}-\frac{a^{2}}{4}
$$

Comparing with Table 1, one can see that the resulting jerk equation belongs to the class $J D_{2}$ with

$$
k_{1}=-c, k_{2}=-b \text { and } k_{3}=-\frac{a^{2}}{4} .
$$

Theorem 3.2. Genesio system has no equivalent jerky dynamics in the variables $y$ and $z$.

Proof. For a simultaneous existence of jerky dynamics in $y$ and/or $z$ first, the following conditions must be satisfied:

$$
n_{2}(\boldsymbol{x})=n_{2}(y) \text { and } / \text { or } n_{3}(\boldsymbol{x})=n_{3}(z)
$$


For the Equation (16) we have

$$
n_{2}(\boldsymbol{x})=0 \text { and/or } n_{3}(\boldsymbol{x})=x^{2} \neq n_{3}(z) .
$$

Since the second equation in (22) does not hold we can conclude that the Equation (17) cannot have a jerky dynamics in $z$.

From the condition (8) we get $f_{2}(y, z)=x^{2}$ which is absurd. This follows that the Genesio system cannot have a jerky dynamics in $y$ also.

Theorem 3.3. The Genesio system is a Newtonian jerky dynamics.

Proof. The Equation (21) can be put in the form (12)

$$
\dddot{x}+p(x, \dot{x}) \ddot{x}+q(x, \dot{x})=0
$$

where $p(x, \dot{x})=c$ and $q(x, \dot{x})=b \dot{x}-x^{2}+\frac{a^{2}}{4}$. Since both $p$ and $q$ are differentiable and integrable functions of their arguments $x$ and $\dot{x}$ the jerky dynamics (21) is Newtonian by Theorem 2.3.

Corollary 3.1. The Genesio system is not a gradient system.

Proof. From Theorem 3.3 we know that the Genesio system is a Newtonian jerky. Since Newtonian jerky dynamics are not gradient systems by Theorem 2.4, the Genesio system is not a gradient system.

Theorem 3.4. The Genesio system exhibits chaotic solutions for some parameter ranges.

Proof. We can write Equation (21) as

$$
\dddot{x}+c \ddot{x}+b \dot{x}=x^{2}-\frac{a^{2}}{4}
$$

Integration of Equation (23) yields

$$
\ddot{x}+c \dot{x}=\int^{t}\left[x(\tau)^{2}-\frac{a^{2}}{4}\right] \mathrm{d} \tau
$$

The memory term $x^{2}-\frac{a^{2}}{4}$ changes sign as $x$ varies, that is, it is neither positive semi-definite nor negative semi-definite for all $x$. Therefore the Genesio system can have chaotic solutions for some parameter ranges by Theorem 2.5.

\section{Dynamical Properties}

Given the jerky dynamics

$$
\dddot{x}+c \ddot{x}+b \dot{x}-x^{2}+\frac{a^{2}}{4}=0
$$

the equilibria can be found by assuming that it has a fixed point $x^{*} \in \square, \dot{x}^{*}=0, \ddot{x}^{*}=0$, which leads to $J\left(x^{*}, 0,0\right)=x^{2}-\frac{a^{2}}{4}=0$, or $x^{*}= \pm \frac{a}{2}$. So there are two equilibria: $E_{+}\left(\frac{a}{2}, 0,0\right)$ and $E_{-}\left(-\frac{a}{2}, 0,0\right)$. Linearizing
Equation (24) about the equilibrium $E_{+}$provides one real and a pair of complex conjugate eigenvalues along with the following characteristic equation

$$
\lambda^{3}+c \lambda^{2}+b \lambda-a=0
$$

and linearizing the Equation (24) about the other equilibrium $E_{-}$yields the following characteristic equation

$$
\lambda^{3}+c \lambda^{2}+b \lambda+a=0 .
$$

According to Routh-Hurwitz criteria, the equilibrium $E_{+}$is stable (i.e., the real part of all roots $\lambda_{i}(i=1,2,3)$ of Equation (25) are negative) only if the conditions

$$
a<0, b>0, c>0, b c+a>0
$$

are fulfilled. For $b c=-a(a<0, b, c>0)$, the fixed point becomes unstable and the two complex roots of (25) cross the imaginary axes, while the third root remains real and negative. Therefore at $b c=-a$ a stable limit cycle arises via a Hopf bifurcation.

$E_{-}$has the same stability characterization. If

$$
a>0, b>0, c>0, b c-a>0
$$

then Equation (26) satisfies the Routh-Hurwitz criteria and at $b c=a$ a stable limit cycle arises via a Hopf bifurcation.

The volume contraction rate of the Equation (24) is

$$
\Lambda=\partial_{\ddot{x}}\left(-c \ddot{x}-b \dot{x}+x^{2}-\frac{a^{2}}{4}\right)=-c,
$$

i.e., $\frac{1}{V} \frac{\mathrm{d} V}{\mathrm{~d} t}=-c$, which can be solved to yield

$V(t)=V(0) \mathrm{e}^{-c t}$. When $c$ is positive, the jerky dynamics Equation (24) is dissipative with solutions for $t \rightarrow \infty$ that contract at an exponential rate $-c$ onto an attractor of zero volume that may be an equilibrium point, a limit cycle, or a strange attractor. When $c=0, \Lambda$ is zero and the phase space volume conserved and the dynamical system is conservative. When $c$ is negative, $\Lambda$ is positive and the volume expands exponentially fast and there are only unstable fixed points. Therefore the dynamics diverges for $t \rightarrow \infty$ if the initial value does not lie exactly at such unstable set.

The Equation (24) has three free parameters $a, b$ and $c$ and the position of equilibria $E_{+}\left(\frac{a}{2}, 0,0\right), E_{-}\left(-\frac{a}{2}, 0,0\right)$ depends on the parameter $a$. To get the parameter independent equilibria we use the transformation

$$
\bar{x}=\frac{2}{a} x, \bar{t}=c t
$$

for $x$ and $t$ yielding the new quantities $\bar{x}$ and $t$. With the substitution of Equation (29), Equation (24) becomes

$$
\dddot{\bar{x}}=-\ddot{\bar{x}}-\frac{b}{c^{2}} \dot{\bar{x}}-\left(-\frac{a}{2 c^{3}}\right)\left(\bar{x}^{2}-1\right)
$$


introducing $\alpha=\frac{b}{c^{2}}, \quad \beta=-\frac{a}{2 c^{3}}$ as new parameters and dropping the overbars we write

$$
\dddot{x}=-\ddot{x}-\alpha \dot{x}-\beta\left(x^{2}-1\right) .
$$

Equation (30) possesses two stationary points $x^{*}= \pm 1$, $\dot{x}^{*}=0, \ddot{x}^{*}=0$. Analyzing their stability leads to the characteristic equation

$$
\lambda^{3}+\lambda^{2}+\alpha \lambda+\mp 2 \beta=0 .
$$

It follows that $x^{*}=1$ is stable only for $\alpha>0$, $\beta>0$ and $\alpha>2 \beta$ and it becomes unstable at the line $\alpha=2 \beta(\alpha>0, \beta>0)$ via a Hopf bifurcation. Similarly, $x^{*}=-1$ is stable only for $\alpha>0, \beta<0$ and $\alpha>-2 \beta$ and it becomes unstable at the line $\alpha=-2 \beta(\alpha>0, \beta<0)$ via a Hopf bifurcation. These stability properties of fixed points also reflect the symmetry of the Genesio system.

Equation (30) is invariant under $x \rightarrow-x$ and $\beta \rightarrow-\beta$. Therefore, knowing the solution of $x(t)$ of Equation (30) for a certain value of parameter $\beta$ and certain initial values, the dynamics of the corresponding sign inverted $\beta$ and initial values is given by $-x(t)$.

Summarizing the results, we conclude that possibly interesting dynamics of the Genesio system (17) is described by Equation (30). Due to the discussed symmetry of this equation, we need only to consider initial values close to one of the two stationary points. Then the most interesting region of the $(\alpha, \beta)$-parameter plane is the one for $\alpha<2 \beta$ and positive $\alpha$. From the studies in $[11,15,16]$, we also know that this parameter region contains homoclinic orbits of the other stationary point $x^{*}=-1, \dot{x}^{*}=0, \ddot{x}^{*}=0$, which here is also a saddlefocus.

\section{Numerical Results}

Besides the local stability and Hopf bifurcation analysis of Equation (30), we also computed the set of all Lyapunov exponents for different values of parameters $\alpha$ and $\beta$ and use to determine and classify the long-time dynamics of the Genesio system. Numerical calculations are performed using Mathematica and iDMC softwares, and RKF45 and RK2Imp are used as numerical algorithms with step size 0.001 . The initial values are chosen as $x^{*}=1.005, \dot{x}^{*}=0.05, \ddot{x}^{*}=0.05$ which are close to the fixed point $x^{*}=1, \dot{x}^{*}=0, \ddot{x}^{*}=0$.

For the parameter regions $\alpha<0$ or $\beta<0$, no bounded solutions have been found. This suggests that the Genesio system does probably not possess at all a stable attractor in these regions. For the region $\alpha>0$ and $\beta>0$ the resulting Lyapunov spectra are shown in Figure 1. The fixed point domain is followed by a large limit cycle region and a structured chaotic region. However the chaotic region is not present for $\alpha \leq 1.6$. At
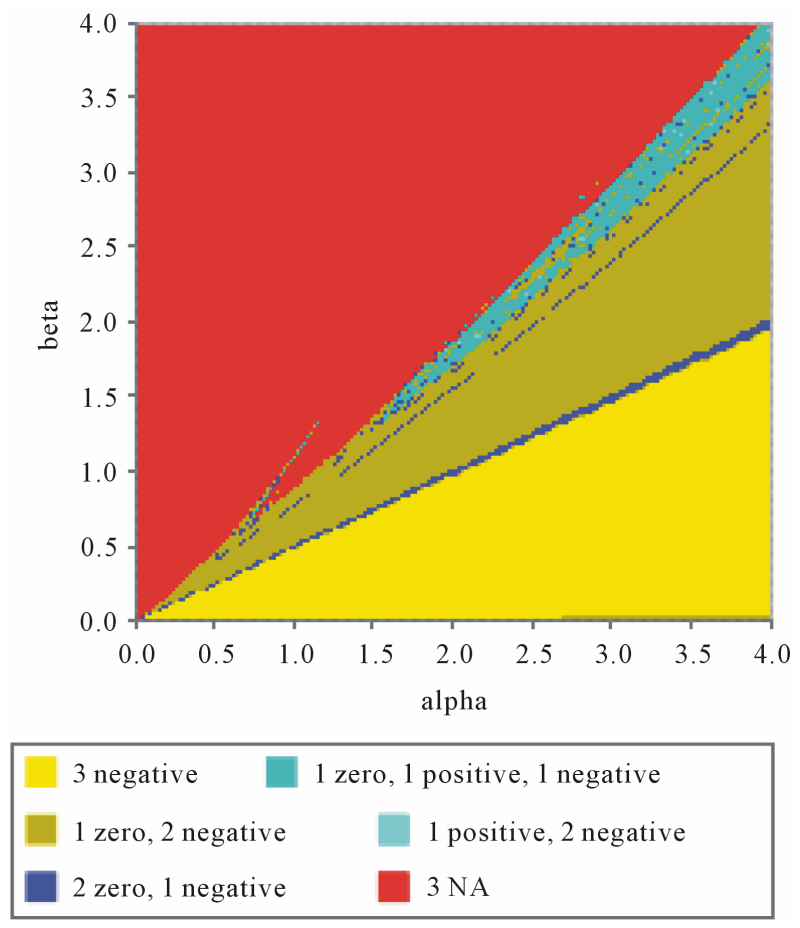

Figure 1. Lyapunov spectra for the jerky dynamics Equation (30).

$\alpha \approx 1.6$ its boundary is formed by two tongues that reach into the limit cycle domain. For smaller parameter values one only finds chaotic points at $\alpha \approx 0.75$ and $\beta \approx 0.71$. Moreover there are islands with bounded dynamics (limit cycles and strange attractors) located within the diverging region.

In Figure 2(a), we have shown the bifurcation diagram which is the plot of successive maxima of the long time evolution of $x(t)$ as a function of system parameter $\beta$ for fixed value of the parameter $\alpha=3.5$. By Figure 2(a), it becomes clear that the jerk dynamical system Equation (30) having quadratic nonlinearity shows the chaos with a cascade of period-doubling bifurcations which is initiated by a supercritical Hopf bifurcation at the line $\alpha=2 \beta$. Therefore the limit cycle domain that follows the fixed point region $(1.5<\beta<1.75)$, consists of periodic attractors with period $2^{n}(n \in N), 1.75<\beta$ $<3.142$.

This region consists of an infinite series of perioddoubling bifurcations. It also contains many narrow windows, which are called limit cycle windows. As $\beta$ is further increased the limit cycle windows break down and eventually disappear. In Figure 2(b), the Lyapunov exponents as a function of the parameter $\beta$ is for the same range as that of the bifurcation plot. In Figure 2(c), the Lyapunov dimension is for the same range of $\beta$ as in Figures 2(a) and (b). It can be clearly seen that all the three frames have one-to-one correspondence with each other, i.e., we observe that for the values of parameter $\beta$ 


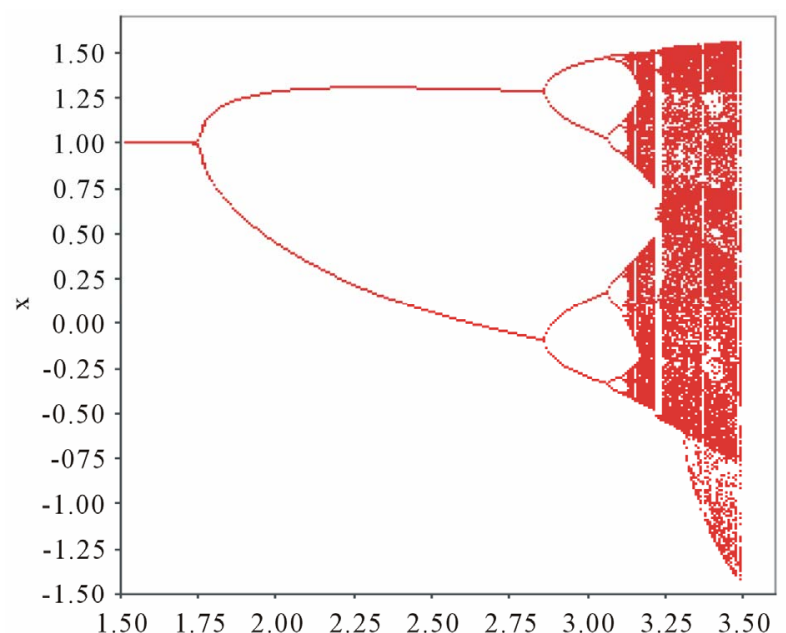

(a)

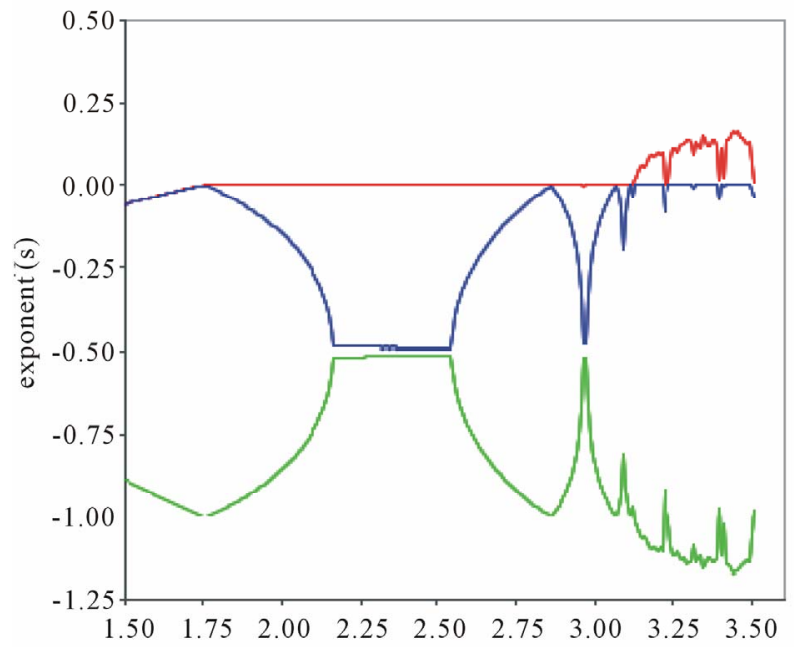

(b)

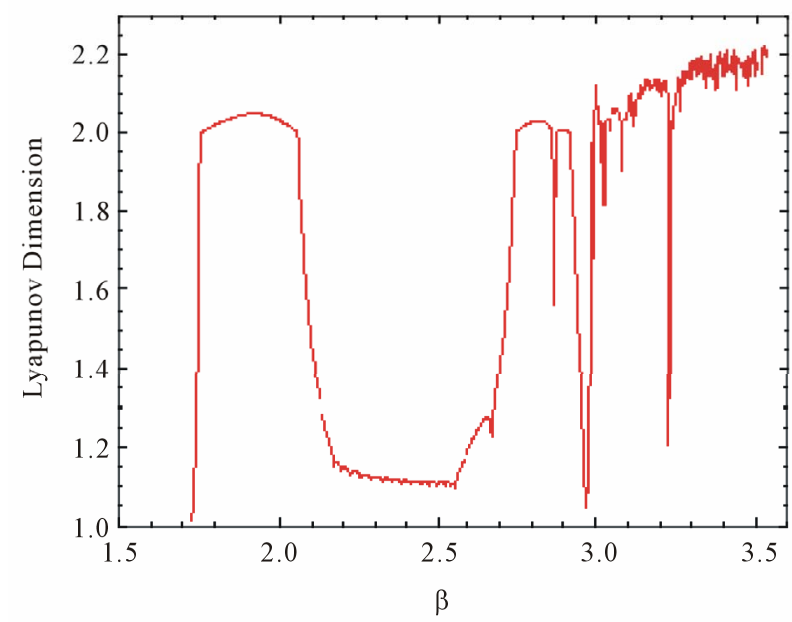

(c)

Figure 2. Behavior of the jerk dynamical system having quadratic nonlinearity Equation (30) for a fixed value of the parameter $\alpha=3.5$. (a) The bifurcation diagram; (b) The Lyapunov exponents; (c) The Lyapunov dimension of the attractor. where the bifurcation diagram shows the limit cycle solutions, the largest Lyapunov exponent is negative as well as the dimension of the attractor is two. However, for the values of the parameter $\beta$ where the bifurcation plot shows the existence of aperiodic behavior (chaotic), the largest Lyapunov exponent is positive as well as the dimension of the attractor is a non-integer 2.15119 between two and three for the parameter values $\alpha=3.5$ and $\beta=3.46$.

For a computed value of $\beta$, we record the successive local maxima of $x(t)$ for a trajectory on the strange attractor. Figure 3 shows $x_{n+1}$ vs $x_{n}$, where $x_{n}$ denotes the $n$th local maximum. The data points for all nearly on a one-dimensional curve. These one-dimensional maps are obtained to compare the different dynamics on a dynamical system. Such maps with parabola like maxima are well-known for the generation of the chaotic solution through period-doubling route and it gives us a clue the route to chaos in the jerk dynamical systems under consideration.

Figure 4 shows two-dimensional projections of the system's attractor for different values of $\beta$ (with $\alpha=3.5$ held fixed). At $\beta=2.5$ the attractor is a stable limit cycle. As $\beta$ is decreased to 3.0 , the limit cycle goes around twice before closing, and its period is approximately twice that of the original cycle. This is what period-doubling looks like in a continuous-time system. In fact, somewhere between $\beta=2.5$ and 3.0, a perioddoubling bifurcation of cycles must have occurred. Another period-doubling bifurcation creates the four-loop cycle shown at $\beta=3.1$. After an infinite cascade of further period-doublings, we obtain the strange attractor at $\beta=3.46$.

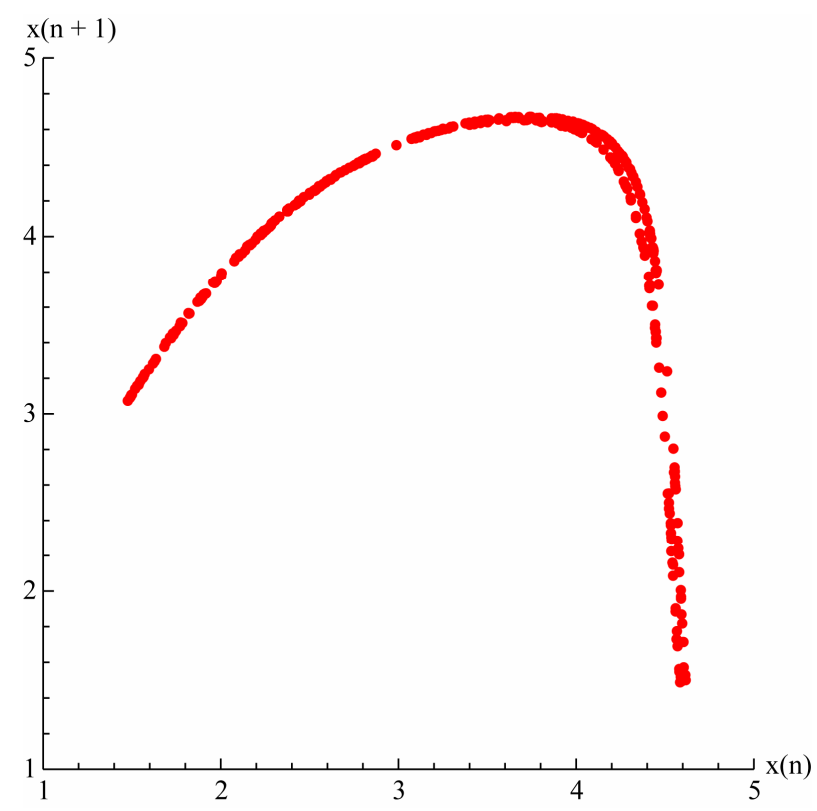

Figure 3. First return map. 


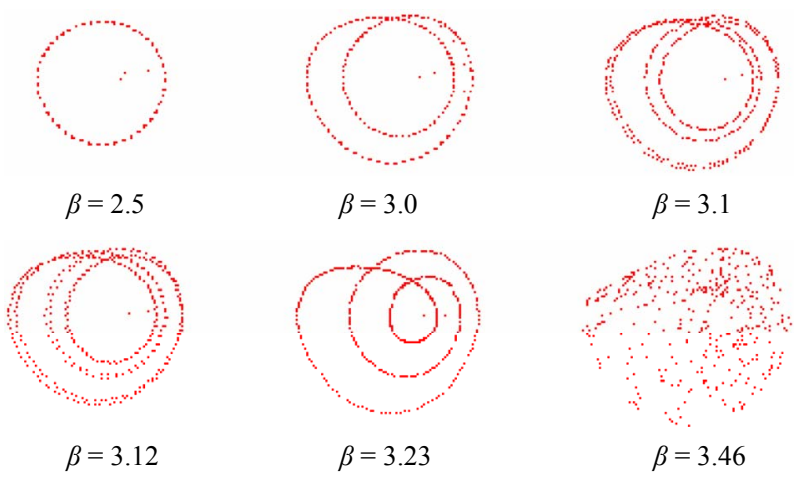

Figure 4. Two-dimensional projections of the attractor of the Equation (30) for different values of $\beta$.

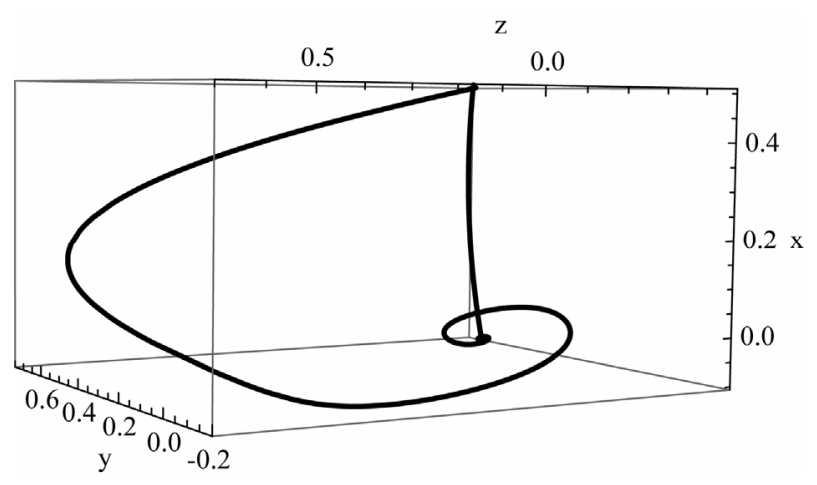

Figure 5. Homoclinic orbit of the Equation (30).

The underlying variety of dynamical behavior can be related to the occurrence of homoclinic orbits as shown in Eichhorn et al. [11] and Glendinning and Sparrow, [15]. In fact, with a numerical search we have been able to detect homoclinic orbits as depicted in Figure 5. Hence the dynamics of the Genesio system is likely determined by the interactions between the stable and unstable manifolds of the single stationary point leading, finally, to homoclinicity.

\section{Discussion}

In this paper, we showed that Genesio system is one of the functionally simplest polynomial classes of jerky dynamics, $J D_{2}$ and it is a Newtonian jerky dynamics. We also investigated some aspects of the dynamical properties of the Genesio system and we have found for this system there are not only few parameters but wide ranges of parameter values that lead to chaotic long time dynamics. Moreover, also large parameter regions are present where the long time attractor of the dynamics consists of stable limit cycles. The route to chaos is determined by a period doubling cascade (for appropriately varied system parameters) that is initiated by a Hopf bifurcation. Varying the initial conditions, we are also able to detect several coexisting stable attractors. Therefore, despite its functional simplicity, the Genesio system shows a rich diversity of dynamical behavior.

We have seen that the complexity and diversity of the dynamics of Equation (30) is associated with the appearance of various homoclinic orbits as in the works of Eichhorn et al. [11], Glendinning and Sparrow [15] and Arneodo et al. [16].

\section{REFERENCES}

[1] S. H. Schot, "Jerk: The Time Derivative of Change of Acceleration," American Journal of Physics, Vol. 46, No. 11, 1978, pp. 1090-1094. doi:10.1119/1.11504

[2] H. P. W. Gottlieb, "Question \#38. What Is the Simplest Jerk Function That Gives Chaos?" American Journal of Physics, Vol. 64, No. 5, 1996, p. 525. doi:10.1119/1.18276

[3] J. C. Sprott, "Some Simple Chaotic Jerk Functions," American Journal of Physics, Vol. 65, No. 6, 1997, pp. 537-543. doi:10.1119/1.18585

[4] J. C. Sprott, "Simplest Dissipative Chaotic Flow," Physics Letters A, Vol. 228, No. 4-5, 1997, pp. 271-274. doi:10.1016/S0375-9601(97)00088-1

[5] S. J. Linz, "Nonlinear Dynamics and Jerky Motion," American Journal of Physics, Vol. 65, No. 6, 1997, pp. 523-526. doi:10.1119/1.18594

[6] S. J. Linz, "Newtonian Jerky Dynamics: Some General Properties," American Journal of Physics, Vol. 66, No. 12, 1998, pp. 1109-1114. doi:10.1119/1.19052

[7] A. Maccari, "The Non-Local Oscillator," Nuovo Cimento $B$, Vol. 111, No. 8, 1996, pp. 917-930. doi:10.1007/BF02743288

[8] R. Eichhorn, S. J. Linz and P. Hanggi, "Transformations of Nonlinear Dynamical Systems to Jerky Motion and Its Application to Minimal Chaotic Flows," Physical Review E, Vol. 58, No. 6, 1998, pp. 7151-7164. doi:10.1103/PhysRevE.58.7151

[9] C. W. Wu, "On Nonlinear Dynamical Systems Topologically Conjugate to Jerky Motion via a Linear Transformation," Physics Letters A, Vol. 296, No. 2-3, 2002, pp. 105-108. doi:10.1016/S0375-9601(02)00267-0

[10] O. E. Rössler, "Continuous Chaos: Four Prototype Equations," Annals of the New York Academy of Sciences, Vol. 316, No. 1, 1979, pp. 376-392. doi:10.1111/j.1749-6632.1979.tb29482.x

[11] R. Eichhorn, S. J. Linz and P. Hanggi, "Simple Polynomial Classes of Chaotic Jerky Dynamics," Chaos, Solitons \& Fractals, Vol. 13, No. 1, 2002, pp. 1-15. doi:10.1016/S0960-0779(00)00237-X

[12] L. Perko, "Differential Equations and Dynamical Systems," Springer-Verlag, New York, 1991. doi:10.1007/978-1-4684-0392-3

[13] J. Hubbard and B. West, "Differential Equations: A Dynamical Systems Approach," Springer-Verlag, New York, 1995. doi:10.1007/978-1-4612-4192-8

[14] R. Genesio and A. Tesi, "Harmonic Balance Methods for the Analysis of Chaotic Dynamics in Nonlinear Systems," Automatica, Vol. 28, No. 3, 1992, pp. 531-548. 
doi:10.1016/0005-1098(92)90177-H

[15] P. Glendinning and C. Sparrow, "Local and Global Behavior near Homoclinic Orbits," Journal of Statistical Physics, Vol. 35, No. 5-6, 1984, pp. 645-696.

doi:10.1007/BF01010828
[16] A. Arneodo, P. H. Coullet, E. A. Spiegel and C. Treser, “Asymptotic Chaos," Physica D, Vol. 14, No. 3, 1985, pp. 327-347. doi:10.1016/0167-2789(85)90093-4 